\title{
Potential gains in reproductive-aged life expectancy if maternal mortality were eradicated from the Kintampo districts of Central Ghana
}

\author{
Sulemana Watara Abubakari ${ }^{*}$ D, Ayaga Agula Bawah², Ernest Obed Nettey', Edward Anane Apraku', \\ Charles Zandoh', Seeba Amenga-Etego ${ }^{1}$, Kwaku Poku Asante ${ }^{1}$, Seth Owusu-Agyei ${ }^{3}$ and Delali Margaret Badasu ${ }^{2}$
}

\begin{abstract}
Background: Almost 99\% of pregnancy or childbirth-related complications globally is estimated to occur in developing regions. Yet, little is known about the demographic impact of maternal causes of death (COD) in lowand middle-income countries. Assuming that critical interventions were implemented such that maternal mortality is eradicated as a major cause of death, how would it translate to improved longevity for reproductive-aged women in the Kintampo districts of Ghana?

Methods: The study used longitudinal health and demographic surveillance data from the Kintampo districts to assess the effect of hypothetically eradicating maternal COD on reproductive-aged life expectancy by applying multiple decrement and associated single decrement life table techniques.

Results: According to the results, on the average, women would have lived an additional 4.4 years in their reproductive age if maternal mortality were eradicated as a cause of death, rising from an average of 28.7 years lived during the 2005-2014 period to 33.1 years assuming that maternal mortality was eradicated. The age patterns of maternal-related mortality and all-cause mortality depict that the maternal-related mortality is different from the all-cause mortality for women of reproductive age.
\end{abstract}

Conclusion: This observation suggests that other COD are competing with maternal mortality among the WRA in the study area and during the study period.

Keywords: Maternal mortality, Causes of death, Reproductive-aged life expectancy, Women of reproductive age

\section{Background}

Women in every country are reported to have a longer life expectancy at birth than men [1]. However, there is little understanding of how maternal mortality influences improvement in women's life expectancy. Understanding the effects of maternal mortality on life expectancy of women is particularly important in low-and middle-income countries (LMICs) where 99\% of maternal deaths occur [1]. Despite the attention that maternal mortality has received over the past three decades, researchers have paid little attention to

\footnotetext{
* Correspondence: abubakari.sulemana@kintampo-hrc.org

${ }^{1}$ Kintampo Health Research Centre, Ghana Health Service, P. O. Box 200,

Kintampo, Ghana

Full list of author information is available at the end of the article
}

the measurement of the demographic impact of maternal mortality in LMICs, especially, in sub-Saharan Africa (SSA) where the burden of maternal mortality is highest.

The majority of the countries in SSA region have maternal mortality ratios (MMR) of over 300 maternal deaths per 100,000 live-births [2, 3]. Maternal mortality in excess of 300 maternal deaths per 100,00 live births is classified as high [3]. SSA, compared to other developing regions of the world, has a very high MMR [4, 5]. It has a point-estimate of 546 compared to 187 in Oceania, 176 in Southern Asia and 110 in South-eastern Asia. Moreover, the lifetime risk of maternal mortality is estimated at 1 in 36 in SSA compared to 1 in 2300 in Eastern Asia and 1 in 1100 in Caucasus and Central

(c) The Author(s). 2019 Open Access This article is distributed under the terms of the Creative Commons Attribution 4.0 International License (http://creativecommons.org/licenses/by/4.0/), which permits unrestricted use, distribution, and 
Asia. In the high-income settings, the lifetime risk is estimated at 1 in 4900 which contrasts sharply with the risk in SSA. Among the 20 countries with the highest maternal mortality ratios, only Afghanistan is not in SSA region [4]. Using the 1990 to 2013 global burden of disease (GBD) data, there would be 184,100 maternal deaths globally in 2030. SSA is projected to account for 38 out of 53 countries that are estimated to have MMR of more than 100 maternal deaths per 100,000 livebirths which fall short of the less than 70 maternal deaths per 100,000 live births SDG 3.1 target [5].

Although efforts intensified to reduce maternal mortality since the launch of the "Safe Motherhood" initiative in the late 1980s, progress has been rather slow, the result being continuous and persistent high levels of mortality during pregnancy or childbirth by women. Questions as to how many woman-lives could be saved during pregnancy or at childbirth remain, particularly in SSA. This study is intended to bridge the gap in the literature by assessing the total number of female person-years that might be saved during the reproductive period if maternal mortality were eradicated from the population of women of reproductive age (WRA).

A longitudinal health and demographic surveillance data from the Kintampo Health Research Centre (KHRC) of Ghana were used. To answer the question "how many woman-lives could be saved if deaths due to pregnancyrelated or childbirth were averted, we used multiple decrement and associated single decrement life table techniques $[6,7]$ to estimate woman-life years saved. We conducted the analysis on the assumption that the mortality conditions for the period 2005-2014 for WRA remained unchanged.

\section{Methods}

\section{Data source}

Data for this study came from the Kintampo Health and Demographic Surveillance System (KHDSS) of KHRC. KHDSS covers the Kintampo districts of the Brong Ahafo Region of Ghana [8-10]. One of the key characteristics of the KHDSS is its longitudinal approach to the measurement of demographic and health variables. Repeated visits by fieldworkers at regular intervals to all residential units in the KHDSS area allow them to collect a prescribed set of attribute data on registered subjects, who are consistently and uniquely identified. All deaths that occur in the study area are captured using death registration forms and information subsequently entered into a death registration database which forms part of the linked database of the KHDSS.

To ascertain the cause of death, a validated verbal autopsy (VA) instrument was used to elicit information on circumstances and conditions that prevailed as observed by the primary caregiver of the deceased, from when the individual fell sick until her death. Verbal autopsy involves interviewing relatives or caregivers who were closely associated with the deceased during the period leading to her death $[7,11]$. Two physicians independently reviewed the VA questionnaire and independently assign a probable cause of death, using the 10th revision of the International Statistical Classification of Diseases and Related Health Problems (ICD-10). If there is agreement between the two independently assigned cause, then it is designated as the cause of death. However, if there is no agreement between the two physicians, a third physician is asked to examine the case and if there is no agreement, then the cause of death for that case is considered unknown or indeterminate $[9,12,13]$.

\section{Data management}

Prior to data processing, data verification and consistency checks were conducted to ensure completeness and consistency. In addition, basic demographic checks such as age-specific mortality rates were conducted to ensure that the mortality profile exhibited by the data is consistent with what is expected of a developing country like Ghana. Data processing was carried out using Microsoft Visual Foxpro (version 9.0). All VA questionnaires were double-entered and verified with an automated range and consistency checks.

\section{Estimation procedure}

This study defines maternal deaths to include only direct or obstetric COD as the death of a woman while pregnant or within 42 days after the termination of the pregnancy, irrespective of the duration and site of the pregnancy. Reproductive-aged life expectancy (RALE) which is estimated in this study refers to the average number of years to be lived by a woman during her reproductive age period (15-49 years), is calculated similar to that of overall life expectancy but limited to the ages from 15 to 49 years, using age 15 as the radix of the life table in this case. RALE is suitable for this study because maternal mortality is conditioned on survival to age 15 and takes into account all causes of mortality $[14,15]$.

The effect of maternal COD on overall mortality was evaluated by estimating the number of person-years that could be saved assuming maternal COD were eradicated as a major cause of death among females in the Kintampo districts. The impact of maternal COD is estimated using multiple-decrement and associated single-decrement lifetable techniques $[6,7,12]$. These methods estimate the net effect of competing risks from different causes. To undertake this analysis, we isolated all deaths due to maternal causes (pregnancy or childbirth related deaths) and lumped all other causes other than the identified maternal deaths into an alternate category. This is important because of the competing risks analysis (maternal versus 
all other causes). Competing risks analysis operates under the assumption that different causes operate independently of each other to affect mortality. An algebraic explication of the estimation procedure as outlined by Bawah and Binka (2007) is shown in the Appendix.

\section{Data limitations}

The influence of mortality from maternal COD may not have been estimated accurately since not all deaths recorded by the KHDSS had successful verbal autopsy interviews. However, this is expected to be self-selected and, therefore, should not have major effects on this study. In addition, a proportion of the cases with successful interviews were coded as "cause of death not determined". This is also expected to be random. Furthermore, there are possibilities of wrongly assigning of COD but again, this is not expected to significantly affect the results since they are very likely to be self-selected. Moreover, child deaths are more likely to be missed than adults. Babies who die a few days or weeks after delivery are usually not reported by some family members who do not count them as human beings. Individuals considered for this study were all adults who were less likely to have been missed under such circumstances.

\section{Results}

Table 1 presents a general and multiple-decrement life table for WRA in the Kintampo districts from 2005 to 2014. We restricted the life table to WRA because of our interest in computing reproductive life years saved. For the period 2005-2014, a total of 1259 deaths occurred among women in their reproductive ages (15-49 years), of which 74 women were estimated to have died from maternal-related causes as defined earlier. By age group, 116 women died in 15-19 age group, 163 in the 20-24 age group, 213 in the 25-29 age group, and so on from allcause mortality. The distribution of the 74 maternal mortality related deaths by age group within the same age groups were 11 within the age-group 15-19, 14 in the 2024 age group, 25 in the 25-29 age group, 11 in the 30-34 age group, 7 in the 35-39 age group, 4 in the 40-44, and 2 in the 45-49 age group. Constructing a single decrement life table for all deaths irrespective of cause results in a life expectancy of 28.7 for women within the reproductive ages (ages 15-49 years).

To estimate the impact of maternal mortality on overall mortality for women in their reproductive ages, we isolate the 74 deaths due to maternal mortality and estimated a multiple decrement life table to enable us answer the question 'how many women who reached the reproductive age would eventually die from maternal mortality related causes?' So, assuming a hypothetical situation where 100,000 women survived from birth to age 15 and are subjected to the age-specific mortality conditions of the period, about 703 of them would eventually die from maternal mortality by the time they attain age 49 (see third to the last column of Table 1). In Table 2, we estimate the gains in RALE assuming maternal mortality was eradicated. To do this, we used causedeleted life table analysis (Table 2). The resultant RALE is estimated at 33.1, suggesting a gain of 4.4 years, from a reproductive age life expectancy of 28.7 to 33.1 years, during the period.

Table 1 A general and multiple-decrement life table for WRA, KHDSS (2005 to 2014)

\begin{tabular}{|c|c|c|c|c|c|c|c|c|c|c|c|c|c|c|c|c|}
\hline Age $x$ & PYO & $\mathrm{D}^{\text {All }}$ & $\mathrm{D}^{\mathrm{MM}}$ & $n a_{x}$ & ${ }_{n} m_{x}$ & $n q_{x}$ & ${ }_{n} P_{x}$ & $I_{x}$ & ${ }_{n} d_{x}$ & $n L_{x}$ & $\mathrm{~T}_{\mathrm{x}}$ & $e_{x}$ & $n q_{x}^{M M}$ & ${ }_{n} d_{x}{ }^{M M}$ & $I_{x}^{M M}$ & ${ }_{n} m_{x}{ }^{M M}$ \\
\hline 15-19 & 70,605 & 116 & 11 & 2.478 & 0.0016 & 0.0082 & 0.9918 & 100,000 & 818 & 497,937 & $2,865,517$ & 28.7 & 0.0008 & 78 & 703 & 0.0002 \\
\hline $20-24$ & 60,178 & 163 & 14 & 2.711 & 0.0027 & 0.0135 & 0.9865 & 99,182 & 1335 & 492,853 & $2,367,580$ & 23.9 & 0.0012 & 115 & 626 & 0.0002 \\
\hline $25-29$ & 52,484 & 213 & 25 & 2.605 & 0.0041 & 0.0201 & 0.9799 & 97,847 & 1966 & 484,525 & $1,874,727$ & 19.2 & 0.0024 & 231 & 511 & 0.0005 \\
\hline $30-34$ & 46,333 & 222 & 11 & 2.565 & 0.0048 & 0.0237 & 0.9763 & 95,881 & 2271 & 473,874 & $1,390,202$ & 14.5 & 0.0012 & 113 & 280 & 0.0002 \\
\hline $35-39$ & 39,707 & 227 & 7 & 2.534 & 0.0057 & 0.0282 & 0.9718 & 93,610 & 2639 & 461,544 & 916,327 & 9.8 & 0.0009 & 81 & 168 & 0.0002 \\
\hline $40-44$ & 33,456 & 178 & 4 & 2.470 & 0.0053 & 0.0262 & 0.9738 & 90,971 & 2388 & 448,816 & 454,784 & 5.0 & 0.0006 & 54 & 86 & 0.0001 \\
\hline $45-49$ & 26,742 & 140 & 2 & 2.606 & 0.0052 & 0.0259 & 0.9741 & 88,584 & 2290 & 5968 & 5968 & 0.1 & 0.0004 & 33 & 33 & 0.0001 \\
\hline Total & 329,505 & 1259 & 74 & - & - & - & - & - & - & - & - & - & - & 703 & - & - \\
\hline
\end{tabular}

Data source: Kintampo HDSS (2005-2014)

Notes:

PYO = Person years of observation

$\mathrm{MM}=$ Maternal causes of death

${ }_{n} a_{x}=$ Average number of person-years lived in the interval by those who have died in the interval

${ }_{n} \mathrm{~m}_{\mathrm{x}}=$ Mortality rate for people in age group $\mathrm{x}$ to $\mathrm{x}+\mathrm{n}$

$n q_{x}=$ Probability of dying between ages $x$ and $x+n$

${ }_{n} p_{x}=$ Probability of surviving between ages $x$ and $x+n$

$I_{x}=$ Number surviving at each age

${ }_{n} d_{x}=$ Number of deaths between ages $x$ and $x+n$

${ }_{n} L_{x}=$ Person-years lived between ages $x$ and $x+n$

$T_{x}=$ Person-years lived beyond age $x$

$\mathrm{e}_{\mathrm{x}}=$ Life expectancy at age $\mathrm{x}$ 
Table 2 Associated single-decrement life table for COD other than maternal causes for KHDSS from 2005 to 2014

\begin{tabular}{llllllllllllll}
\hline Age $x$ & $I_{x}$ & ${ }_{n} P_{x}$ & $R^{-M M}$ & $P^{-M M}$ & $I_{x}^{-M M}$ & ${ }_{n} q_{x}{ }^{-M M}$ & ${ }_{n} d_{x}{ }^{-M M}$ & ${ }_{n} q_{x} /{ }_{n} q_{x}{ }^{-M M}$ & ${ }_{n} a_{x}{ }^{-M M}$ & ${ }_{n} m_{x}{ }^{-M M}$ & ${ }_{n} L_{x}{ }^{-M M}$ & $T_{x}{ }^{-M M}$ & $e_{x}{ }^{-M M}$ \\
\hline $15-19$ & 100,000 & 0.9918 & 0.9052 & 0.9926 & 100,000 & 0.0074 & 741 & 1.1043 & 2.4969 & 0.0015 & 498,146 & $3,306,877$ & 33.1 \\
$20-24$ & 99,182 & 0.9865 & 0.9141 & 0.9877 & 99,259 & 0.0123 & 1222 & 1.0933 & 2.6705 & 0.0025 & 493,450 & $2,808,731$ & 28.3 \\
$25-29$ & 97,847 & 0.9799 & 0.8826 & 0.9822 & 98,037 & 0.0178 & 1741 & 1.1316 & 2.6133 & 0.0036 & 486,031 & $2,315,282$ & 23.6 \\
$30-34$ & 95,881 & 0.9763 & 0.9505 & 0.9775 & 96,296 & 0.0225 & 2169 & 1.0515 & 2.5799 & 0.0046 & 476,233 & $1,829,251$ & 19.0 \\
$35-39$ & 93,610 & 0.9718 & 0.9692 & 0.9727 & 94,128 & 0.0273 & 2573 & 1.0314 & 2.5147 & 0.0055 & 464,244 & $1,353,018$ & 14.4 \\
$40-44$ & 90,971 & 0.9738 & 0.9775 & 0.9743 & 91,555 & 0.0257 & 2350 & 1.0227 & 2.4892 & 0.0052 & 451,875 & 888,774 & 9.7 \\
45-49 & 88,584 & 0.9741 & 0.9857 & 0.9745 & 89,205 & 0.0255 & 2274 & 1.0143 & 0.9857 & 0.0052 & 436,899 & 436,899 & 4.9
\end{tabular}

Data source: Kintampo HDSS (2005-2014)

Note:

$\mathrm{MM}=$ Maternal causes of death

$\mathrm{R}^{-\mathrm{MM}}=$ the proportion of deaths due to all causes other than maternal causes

${ }_{n} a_{x}=$ Average number of person-years lived in the interval by those who have died in the interval

${ }_{n} m_{x}=$ Mortality rate for people in age group $x$ to $x+n$

$n q_{x}=$ Probability of dying between ages $x$ and $x+n$

${ }_{n} p_{x}=$ Probability of surviving between ages $x$ and $x+n$

$\mathrm{I}_{\mathrm{x}}=$ Number surviving at each age

${ }_{n} d_{x}=$ Number of deaths between ages $x$ and $x+n$

${ }_{n} L_{x}=$ Person-years lived between ages $x$ and $x+n$

$\mathrm{T}_{\mathrm{x}}=$ Person-years lived beyond age $\mathrm{x}$

$\mathrm{e}_{\mathrm{x}}=$ Life expectancy at age $\mathrm{x}$

Figure 1 shows the age pattern of COD for overall mortality and maternal-related mortality for WRA. The age pattern of maternal-related mortality is not similar to that of all-cause mortality for WRA. The maternalrelated mortality peaked at 25-29 years and declined steadily after that while the overall mortality for WRA peaked at 35-39 years and began a downward trend after that but remained stable from age 40 to 49 . This suggests that causes other than maternal-related death are the major COD among the WRA in the study area and during the study period.

Figure 2 shows the RALE at every age when all COD among WRA are put together and the corresponding RALE if maternal mortality were removed. At every age, RALE increased if maternal mortality were averted. The rise in life expectancy is relatively more evident at the older ages of life, when the force of mortality is higher than at other ages.

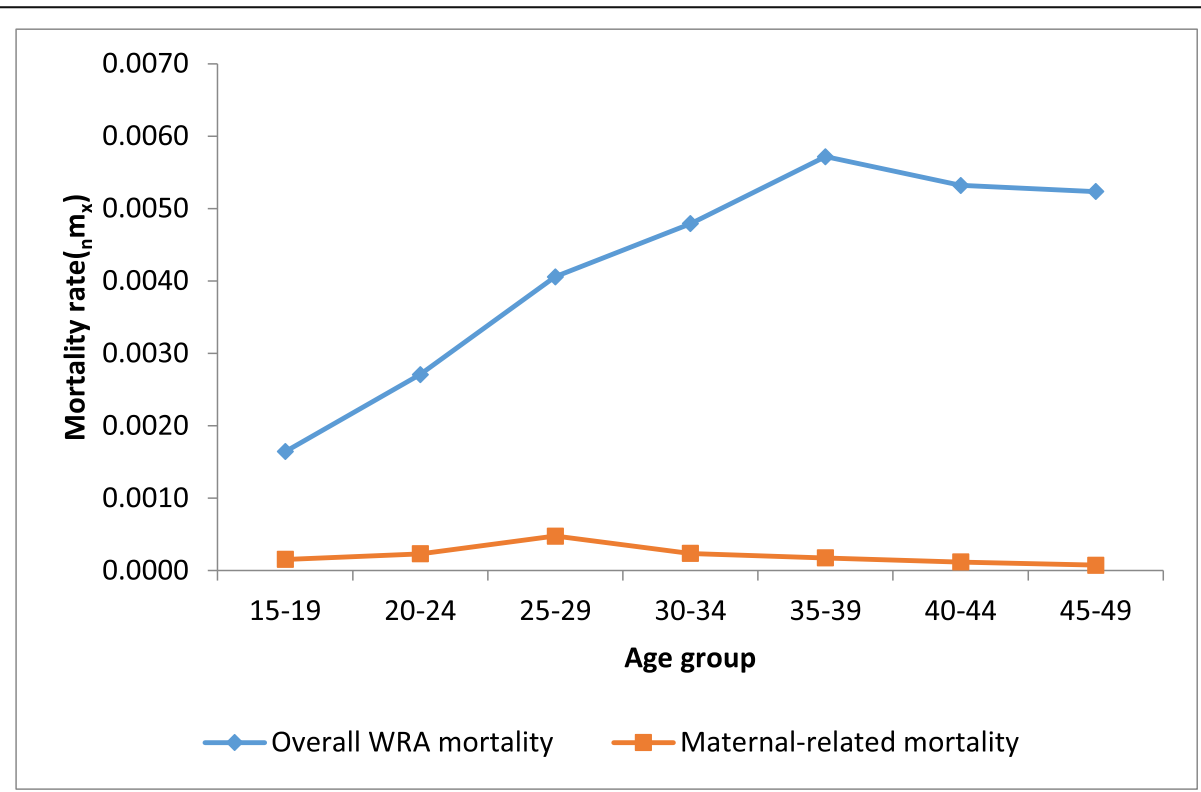

Data source: Kintampo HDSS (2005-2014)

Fig. 1 Age pattern of maternal causes for overall mortality and maternal causes-specific mortality 


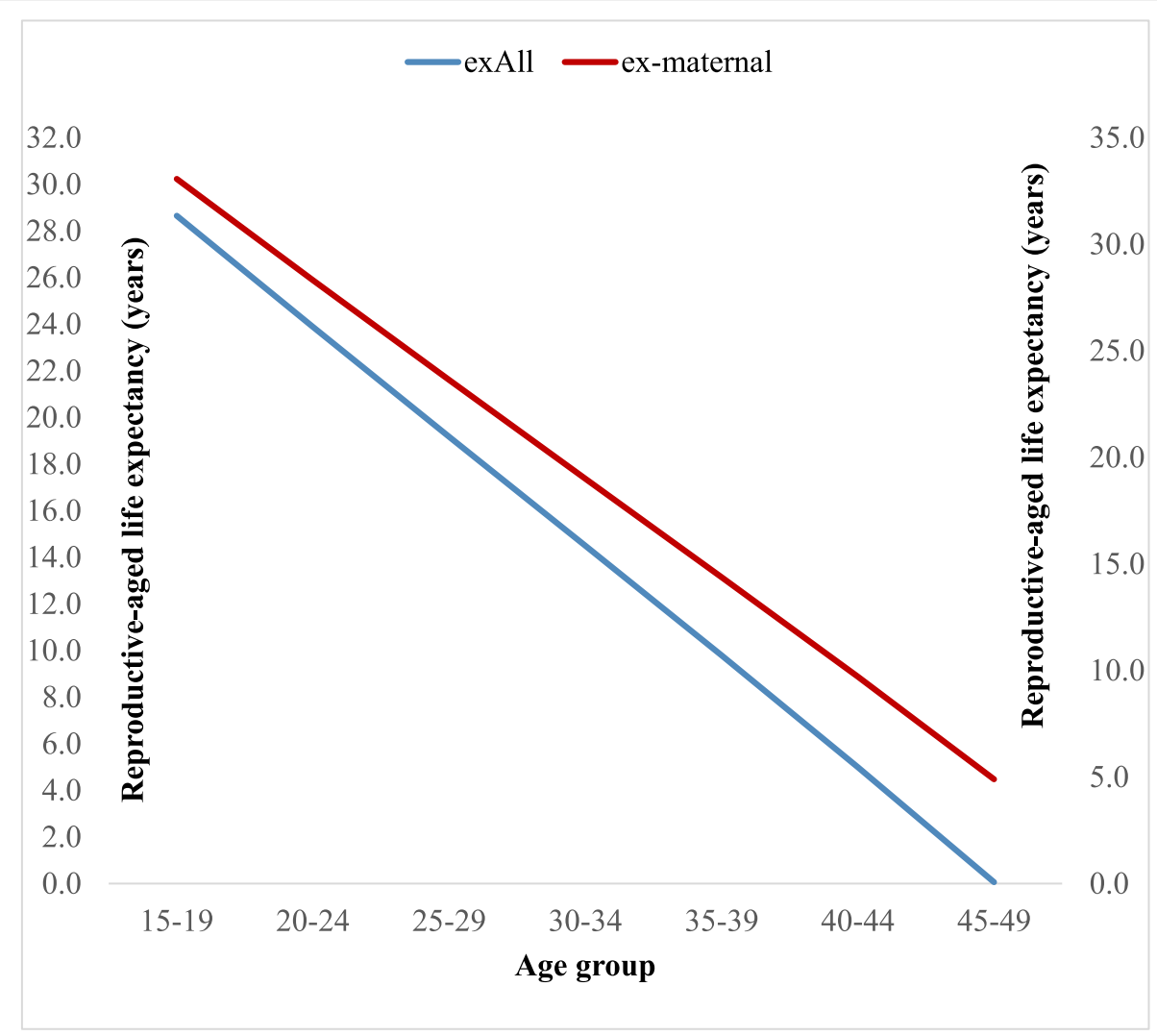

Note: exAll = RALE for all causes of death; ex-maternal = RALE without maternal cause of death

Data source: Kintampo HDSS (2005-2014)

Fig. 2 Comparison of estimated life expectancy for WRA with all-cause mortality and without maternal COD, KHDSS (2005-2014)

\section{Discussions}

This paper sought to estimate potential improvement in RALE if maternal mortality had been eradicated or reduced to insignificant limits such that it is no longer of public health concern in the study area. The results show that the average number of years lived between ages 15 and 49 increased from 28.7 to 33.1 years in the absence of maternal mortality, a probable gain of about 4.4 years. This provides an average annual gain in years of about 0.4 per year over the tenyear study period (2005-2014).

RALE gains of 0.4 years per annum observed in the present study is quite consistent with findings from both the Global North and Global South. For instance, Canudas-Romo et al. (2014) reported an annual gain of 0.5 years due to elimination of maternal mortality in high-income settings during the first half of the twentieth century. Similarly, they reported an annual gain of 0.6 years, assuming maternal mortality was eliminated in low and middle-income countries between 2000 to 2011 [12]. Evidence of consistent reductions in maternal mortality makes the finding in the present study quite plausible [13, 14].
It is documented that maternal mortality declined by 1.5\% per year between 1990 and 2015 globally [3]. The decline was largely as a result of global efforts in the past three decades [15]. Improvement in maternal mortality accelerated after the introduction of MDG 5A in 2000 which aimed to reduce the global MMR by at least $75 \%$ by 2015 [13, 14]. It is observed from the present study that causes other than maternal-related mortality are the major COD among the WRA in the study area and during the study period. This observation is probably due to the current evidence of reduction in maternal mortality as a competing cause of death.

Since the additional survival time of 0.4 years takes place during the most productive ages of women, their contributions to the socio-economic development of families, labour force, communities, districts and the country as a whole would be invaluable if maternal mortality were eradicated. Households that experience a maternal death may suffer reduced economic output due to the loss of mothers and wives in the household. The loss of a mother in the household may result in changes in household duties and management that affect children's schooling, nutrition and supervision [12] and overall care. 
It is important to note that RALE would increase at every age if maternal mortality were eradicated from this population. The results obtained from the study underscore the need for policy and intervention to focus on all WRA and most especially on those who are older since averting maternal deaths disproportionately lead to a greater rise in RALE among the older WRA who experience a higher force of mortality.

Maternal mortality is relatively a rare event compared to the other COD among WRA and yet influences the extent of the gain in RALE. It is reported that in developed countries, maternal mortality is underestimated by about a third. The inaccuracies are much higher in LMICs $[12,16]$. However, wider usage of VA for mundane national health information systems could potentially enhance the availability of essential and reliable data on COD for disease control programmes.

\section{Conclusion}

RALE would have improved among women in the Kintampo districts if maternal mortality was eradicated from this population, given the age-specific mortality rates for the period 2005-2014. The number of years gained would have been quite considerable, an average of about 0.4 years per year given that the average number of years lived from age 15 to 49 would have increased from 28.7 to 33.1 years in the absence of maternal mortality.

The increase in RALE suggested by the results may be achieved eventually if efforts aimed at reducing maternal mortality are enhanced. There is also the need to investigate other COD that might be competing with maternal death as far as the population of WRA in the study area is concerned since the evidence from this analysis points to competition from other COD.

\section{Appendix}

\section{Estimation procedure for multiple decrement and associated single decrement life tables}

In the analysis of causes of death, the force of the mortality function from different causes is additive because disentangling precisely the effects of other causes of death is difficult, especially in settings where precise measurement is not possible. Thus, the sum of the different causes is equal to all causes combined as represented in Eq. (1) thus:

$$
\mu(x)=\sum_{i=1}^{I} \mu_{i}(x)
$$

where $\mu(x)$ is the force of mortality from all causes combined and parameters $\mu_{i}(x)$ refer to the death rate for the $i$ th cause of death. This implies that the rates of decrements are also additive:

$$
{ }_{n} m_{x}=\sum_{i=1}^{i}{ }_{n} m_{x i}
$$

where ${ }_{n} m_{x}$ is the rate of decrement from all causes and ${ }_{n} m_{x i}$ in this case is the rate of decrement from maternal causes of death.

Considering the basic relationship between mortality rates $\left({ }_{n} m_{x}\right)$ and the probability of dying $\left({ }_{n} q_{x}\right)$ as shown in the conventional life table, the transformation of the rates to probabilities of dying is shown in the following equation as:

$$
{ }_{n} q_{x}=\frac{n_{n} m_{x}}{1+\left(n-{ }_{n} a_{x}\right)_{n} m_{x}},
$$

where ${ }_{n} a_{x}$ is defined as the average number of personyears lived in the interval $x$ to $x+n$ by those who died in the interval. This relationship extends to multipledecrement processes as follows:

$$
{ }_{n} q_{x i}=\frac{n_{n} m_{x i}}{1+\left(n-{ }_{n} a_{x}\right)\left({ }_{n} m_{x i}+{ }_{n} m_{x,-1}\right)},
$$

where ${ }_{n} m_{x i}$ and ${ }_{n} m_{x,-i}$ represent decrement rates from maternal and all other causes other than maternal combined, respectively. Data concerning the causes of death by age and the corresponding number of person-years by the same sub-categories define the probabilities of dying at each age $\left({ }_{n} q_{x}\right)$, by cause of death. However, obtaining the ${ }_{n} a_{x}$ values is often difficult. Therefore, different techniques are employed to estimate the ${ }_{n} a_{x}$ values. First, it is assumed that those who died in the interval on average lived halfway through the interval. Based on this assumption, an initial value of 2.5 is adopted for all age groups with an interval of 5 years. For the younger than 1-year and 1-4-year age groups, a procedure suggested by Coale and Demeny is adopted (Bawah and Binka, 2007).

Using the ${ }_{n} a_{x}$ values of 2.5 in the ${ }_{n} m_{x} \rightarrow{ }_{n} q_{x}$ conversion formula, ${ }_{n} q_{x}$ values are estimated first and the values are used to obtain $n d x$ (the number of deaths between age $x$ and $x+n$ ) in a life table. These ${ }_{n} d_{x}$ estimates are plugged into the iteration formula below to obtain new sets of ${ }_{n} a_{x}$ values. These values are subsequently re-introduced into the ${ }_{n} m_{x} \rightarrow{ }_{n} q_{x}$ conversion formula to re-estimate new ${ }_{n} d_{x}$ values, which are re-introduced in the iteration formula to obtain a new set of ${ }_{n} a_{x}$ values. This process is repeated until stable estimates of ${ }_{n} a_{x}$ are achieved (Bawah and Binka, 2007). The iteration equation used is specified as follows: 


$$
{ }_{n} a_{x}=\frac{-\frac{n}{24}{ }_{n} d_{x-n}+\frac{n}{2}{ }_{n} d_{x}+\frac{n}{24}{ }_{n} d_{x+n}}{{ }_{n} d_{x}} .
$$

The stable ${ }_{n} a_{x}$ values then are used to generate a life table for females in the Kintampo HDSS area through the basic ${ }_{n} m_{x} \rightarrow{ }_{n} q_{x}$ conversion formula. With the overall life table generated, the probability of dying from maternal causes of death $\left({ }_{n} q_{x i}\right)$ is estimated, by applying the proportion of deaths that are due to maternal causes of death to the overall probabilities of dying for each age, ${ }_{n} q_{x}$, as follows:

$$
{ }_{n} q_{x i}={ }_{n} q_{x} \cdot \frac{{ }_{n} D_{x i}}{{ }_{n} D_{x}}
$$

where ${ }_{n} q_{x i}$ and ${ }_{n} D_{x i}$ represent the probability of dying from maternal causes of death and the observed number of deaths from maternal causes of death, respectively. The above relationship assumes that the observed death rates for maternal causes of death $\left({ }_{n} M_{x i}\right)$ are equal to the life-table death rates for maternal causes of death $\left({ }_{n} m_{x i}\right)$, that is, ${ }_{n} M_{x i}={ }_{n} m_{x i}$.

Estimating the contribution of mortality from maternal causes of death to overall mortality also allow to estimate the effect of eliminating maternal causes of death through "cause-deleted" lifetable analysis (Bawah and Binka, 2007). If maternal-related mortality were eliminated as a cause of death, survival at age interval $x$ to $x+n$, will be represented as:

$$
{ }_{n} P_{x,-i}={ }_{n} p_{x}\left(\frac{{ }_{n} D_{x i}}{{ }_{n} D_{x}}\right) .
$$

The approach described above assumes that the force of mortality function from each cause is proportional to all causes combined in the interval $x$ to $x+n$ and constant throughout the interval (Arriaga, 1984). The ${ }_{n} a_{x}$ values for the associated single decrement life table were obtained using the following formula for all age groups except the first two and the last:

$$
{ }_{n} a_{x,-1}=n+R^{i} \frac{{ }_{n} q_{x}}{{ }_{n} q_{x,-1}}\left({ }_{n} a_{x}-n\right),
$$

where ${ }_{n} a_{x_{0}-i}$ refers to the average number of personyears lived by those dying in the interval from all causes other than maternal-related death, and $R i$ represents the proportion of deaths due to maternal-related mortality. For the other age groups, the iteration procedure used for estimating the ${ }_{n} a_{x}$ values in the parent life table is used.

\section{Abbreviations}

COD: Causes of Death; GBD: Global Burden of Disease; ICD-10: the 10th revision of the International Statistical Classification of Diseases and Related Health Problems; IEC: Institutional Ethics Committee; KHDSS: Kintampo Health and Demographic Surveillance System; KHRC: Kintampo Health Research Centre; LMICs: Low- and Middle-Income Countries; MDG: Millennium Development Goal; MMR: Maternal Mortality Ratio;
PCVA: Physician Coding Verbal Autopsy; RALE: Reproductive-aged Life Expectancy; SDGs: Sustainable Development Goals; SSA: Sub-Saharan Africa; UNFPA: United Nations Population Fund; UNICEF: United Nations Children's Emergency Fund; VA: Verbal Autopsy; WHO: World Health Organization; WRA: Women of Reproductive Age

\section{Acknowledgments}

We are grateful to the Director and the management of KHRC as well as the Ghana Health Service for allowing the use of the KHDSS data. The study team wishes to acknowledge useful comments from KHRC staff, and the Faculty and students of Regional Institute for Population Studies, University of Ghana. Our appreciation goes to Dr. Cheikh Mbacke, international public health Consultant and former Senior Advisor to Rockefeller Foundation and William \& Flora Hewlett Foundation for providing useful suggestions for the final version. Also, our sincere gratitude goes to Professor Samuel N. A. Codjoe, Director of Regional Institute for Population Studies for his comments on earlier drafts as well as the community for their forbearance and allowing KHRC to collect data from them.

\section{Authours' contribution}

SWA drafted the manuscript. SWA, AAB and DMB designed the study. SWA EON, EAA, CZ, SAE, SOA and KPA were responsible for the conduct of the study. SWA, AAB, DMB, EON, SOA and KPA participated in the statistical analyses, interpretation and manuscript revisions. All the authors approved the final version and agreed to be accountable for the study.

\section{Funding}

No funding was received for this study.

\section{Availability of data and materials}

The datasets used and/or analysed during the current study are available from the corresponding authour on reasonable request.

\section{Ethics approval and consent to participate}

Written informed consent was obtained from adult participants. In the case of adolescent participants, written consent was obtained from their parents or legally accepted representatives. The KHRC Institutional Ethics Committee reviewed the protocol and all instruments associated with this study as part of the activities of the KHDSS with the following details: Ref: KHRC/IEC/ICF/ 2010-1; FWA: 00011103; and IOR0004854 on the approval certificate.

\section{Consent for publication}

Not applicable.

\section{Competing interests}

The authours declare that they have no competing interests.

\section{Author details}

${ }^{1}$ Kintampo Health Research Centre, Ghana Health Service, P. O. Box 200, Kintampo, Ghana. ${ }^{2}$ Regional, Institute for Population Studies, University of Ghana, P. O. Box LG 96, Legon, Ghana. ${ }^{3}$ Institute of Health Research, University of Health \& Allied sciences, Ho, Volta Region, Ghana.

Received: 10 April 2019 Accepted: 20 September 2019

Published online: 23 October 2019

\section{References}

1. World Health Organization. Maternal Mortality Fact Sheet. World Heal Organ Fact Sheet. 2015;

2. Desai M, Phillips-Howard PA, Odhiambo FO, Katana A, Ouma P, Hamel MJ, et al. An Analysis of Pregnancy-Related Mortality in the KEMRI/CDC Health and Demographic Surveillance System in Western Kenya. PLoS One. 2013; 8(7):e68733.

3. WHO, UNICEF, UNFPA TWB and U. Trends in maternal mortality 1990 to 2015. 2015; Available from: http://datatopics.worldbank.org/hnp/files/Trends in Maternal Mortality 1990 to 2015 full report.PDF. Accessed 31 Oct 2017.

4. Alvarez JL, Gil R, Hernandez V, Gil A. Factors associated with maternal mortality in Sub-Saharan Africa: an ecological study. BMC Public Health [Internet]. 2009;9:462. Available from: http://www.embase.com/search/ results?subaction=viewrecord\&from $=$ export\&id $=L 359630416$. Accessed 31 Oct 2017. 
5. Kassebaum NJ, Bertozzi-villa A, Coggeshall MS, Shackelford KA, Steiner C Heuton KR, et al. Global, regional, and national levels and causes of maternal mortality during 1990 - 2013: a systematic analysis for the Global Burden of Disease Study 2013. Lancet. 2014;384:980.

6. Arriaga EE. Measuring and Explaining the Change in Life Expectancies. Popul (English Ed.). 1984;21(1):83-96.

7. Bawah AA, Binka FN. How Many Years of Life Could Be Saved If Malaria Were Eliminated from a Hyperendemic Area of Northern Ghana? Am J Trop Med Hyg. 2007;77(Suppl 6):145-52.

8. Owusu-Agyei S, Nettey OEA, Zandoh C, Sulemana A, Adda R, AmengaEtego S, et al. Demographic patterns and trends in Central Ghana: baseline indicators from the Kintampo health and demographic surveillance system. Glob Health Action. 2012;5(October 2017):1-11.

9. Nettey OEA, Zandoh C, Sulemana A, Adda R, Owusu-Agyei S. Clustering of childhood mortality in the Kintampo Health and Demographic Surveillance System in Ghana. Glob Health Action [Internet]. 2010;3(1):5258. Available from: https://www.tandfonline.com/doi/full/10.3402/gha.v3i0.5258. Accessed 31 Oct 2017.

10. Abubakari S, Enuameh YA, Mahama E, Nettey OEA, Adjei G, Nuamah GF, et al. Adolescents' willingness and intentions to use contraceptives in rural Ghana. Open J Soc Sci. 2015;03(11):239-49.

11. Ye Y, Wamukoya M, Sankoh O, Ezeh A, Emina JBO, Sankoh O. Health and demographic surveillance systems: a step towards full civil registration and vital statistics system in sub-Sahara Africa? BMC Public Health [Internet]. 2012;12(1):741. Available from: http://www.biomedcentral.com/1471-245 8/12/741\%5Cn, http://www.pubmedcentral.nih.gov/articlerender.fcgi?artid= 3509035\&tool=pmcentrez\&rendertype=abstract. Accessed 31 Oct 2017.

12. Canudas-Romo V, Liu L, Zimmerman L, Ahmed S, Tsui A. Potential gains in reproductive-aged life expectancy by eliminating maternal mortality: a demographic bonus of achieving MDG 5. PLoS One. 2014;9(2):1-7.

13. Alkema L, Chou D, Hogan D, Zhang S, Moller AB, Gemmill A, et al. Global, regional, and national levels and trends in maternal mortality between 1990 and 2015, with scenario-based projections to 2030: a systematic analysis by the un maternal mortality estimation inter-agency group. Lancet [Internet]. 2016;387(10017):462-74 Available from: https://doi.org/10.1016/S01406736(15)00838-7. Accessed 31 Oct 2017

14. Say L, Chou D, Gemmill A, Tuncalp O, Moller AB, Daniels J, et al. global causes of maternal death: a WHO systematic analysis. Lancet Glob Heal. 2014;2(6):323-33.

15. Scrafford CG, Tielsch JM. Maternal deaths account for a small proportion of the Total deaths among reproductive age women. J Womens Heal. 2016; 25(3):242-8.

16. Rosenfield A, Maine D. Maternal mortality-a neglected tragedy. Where is the M in MCH? Lancet. 1985;326(8446):83-5.

\section{Publisher's Note}

Springer Nature remains neutral with regard to jurisdictional claims in published maps and institutional affiliations.

Ready to submit your research? Choose BMC and benefit from:

- fast, convenient online submission

- thorough peer review by experienced researchers in your field

- rapid publication on acceptance

- support for research data, including large and complex data types

- gold Open Access which fosters wider collaboration and increased citations

- maximum visibility for your research: over $100 \mathrm{M}$ website views per year

At $\mathrm{BMC}$, research is always in progress.

Learn more biomedcentral.com/submissions 